\title{
Parameter study for a slatless 2D high-lift airfoil with active separation control using a URANS approach
}

\author{
V. CIOBACA
}

DLR, Lilienthalplatz 7,38108, Braunschweig, Germany

\begin{abstract}
Summary
The numerical investigations described in the present paper deal with active flow separation control for a 2D, 2-element high-lift airfoil. Here, the aim of the flow control application is to actively reduce or eliminate a large flow separation on the flap by a periodic excitation mechanism, known as pulsed blowing. This study explores the resulting effects of the flow control application on the global aerodynamic coefficients and, beyond this, by the analysis of the resulting loads variation by specific actuation parameters. For enabling the extrapolation towards flight Reynolds numbers the URANS computations are addressed at both low speed atmospheric windtunnel conditions $\left(\operatorname{Re} \approx 2 \times 10^{6}\right.$.) as well as for higher Reynolds number comparable to flight conditions $\left(\mathrm{Re} \approx 7 \times 10^{6}\right)$.
\end{abstract}

\section{Introduction}

High efficiency, reduced complexity, low weight and low noise become more and more important for aeronautical industries. Steep landing approaches and reduced system weights are of significant relevance for any novel high lift design. These requirements might be suported by a slatless wing configuration due to the upcoming technologies. To be applicable, the lift loss by omitting the slat must be recovered. The solutions discussed nowadays are more complex trailing edge devices and active flow separation control.

In the past many experimental investigations were carried out at Technical Universtity Berlin (TUB) for active flap separation control through periodic excitation. Addressed to various high-lift configurations from very generic [1] up to complex 3D Wing Body setups [2] the experiments with the addition of momentum in an oscillatory manner indicated promising and reliable results. This active flow control (AFC) technique is here the subject of numerical investigations and is strongly connected with the activities conducted at DLR within the EUproject AVERT, Aerodynamic Validation of Emission Reduction Technologies [3].

In contrast to a steady, tangential blowing for the separation control the pulsed blowing is considered to be vastly more energy-efficient, with savings of even one order of magnitude for the blowing momentum coefficient required by a specific performance increment $[4,5]$. In the case of periodic excitation large coherent 
structures are generated, thereby transferring high momentum fluid to the surface, thus being characterized as an unsteady flow.

In our days the numerical applications for active separation control through periodic excitation still require experimental data for the validation and the verification of the numerical approaches. Not only the flow complexity given the AFC application, but often the sensitivity to inflow parameters (e.g the description of the flow separation regions; the actuation parameters at the slot-exit) shows us the difficulty to quantify the state of the art for such URANS simulations in comparison to the experimental results. For this reason, here the numerical finding will be compared with experimental results when available, for the unperturbed flow as well as with AFC.

\section{Geometry overview}

The geometry of interest is the DLR F15 airfoil in a 2D, 2-element, high lift configuration (wing and trailing edge flap), figure 1. The cruise airfoil contour and the corresponding high lift devices originate as a section of a 3D wing design for a research configuration of a single-aisle aircraft. This airfoil is used intensively for about 5 years at DLR for high-lift investigations, e.g. flow control technologies [6], noise reduction techniques [7]. The flight conditions for this airfoil section are the Mach number $M=0.15$, and the Reynolds number $R e=7 \times 10^{6}$ based on the design point of the $3 \mathrm{D}$ wing $\left(30^{\circ}\right.$ sweep) with the corresponding backwardstransformations. In order to illustrate the capabilities of the pulsed blowing as active separation control technique an "artificial" flap setup was investigated, flap -deflection $\delta_{\mathrm{F}}=49^{\circ}$, -gap $\mathrm{g}_{\mathrm{F}} / \mathrm{c}=0.9 \%$ and -overlap ovl $/ \mathrm{F}=2.3 \%$. It was considered the fact that an industry-relevant high lift configuration is designed to have none or only moderate flow separation on the flap depending on the setup of interest (e.g.: take-off, landing), while for AFC research other ("artificial") flap setups with massive separation are relevant as long as a flap shape design dedicated to flow control is not at hand.

The geometrical setup of the actuation is: slot-actuators, of $0.05 \% \mathrm{c}$ in width, located at $20 \% \mathrm{c}_{\mathrm{F}}$ on the flap suction side, and inclined with $45^{\circ}$ downwards as partly used also in [3].

\section{Numerical approach}

The URANS simulations are performed with the compressible solver DLR-TAU code and the 2D computational domain was discretized with the mesh generator Centaursoft (for hybrid grids). As shown in figure 2, the mesh overview indicates the numerical modeling of a portion for the slot-actuator. The actuation boundary condition, at slot's bottom, is of particular interest here. For an inflow boundary 
condition of three-dimensional subsonic flow, according to the characteristic theory four quantities need to be specified by boundary values and the fifth condition is taken from the solution inside the flow domain. Here, we prescribe the conservative variables for density and momentum and use the value of the pressure from the solution inside the domain. Concerning the turbulence modeling the 2-equations Menter SST k- $\omega$ version was applied. All computations are run fully turbulent. For the numerical setting the actuation frequency $\mathrm{f}=100 \mathrm{~Hz}$ $\left(\mathrm{F}^{+}=\mathrm{f}^{*} \mathrm{c}_{\mathrm{F}} / \mathrm{U}_{\infty}=0.32\right)$ guided the length of the dual time step, where $\Delta \mathrm{t}=2.5 \mathrm{e}-05 \mathrm{sec}$. with 500 inner iterations per time step and 400 physical time steps for each actuation cycle.

\section{Results}

First numerical results show the effect of the momentum coefficient variation, $\mathrm{C}_{\mu}=\left(\rho_{\mathrm{j}} * \mathrm{~S}_{\mathrm{j}} * \mathrm{U}_{\mathrm{j}}{ }^{2}\right) /\left(0.5^{*} \rho_{\infty} * \mathrm{~S}_{\mathrm{ref}} * \mathrm{U}_{\infty}{ }^{2}\right)$, for the low Re number test case, Re $\approx 2 \times 10^{6}$ (Figure 3). The computed lift curves reveal the lift enhancement up to the order of $0.5=50$ lift counts (lc) at constant angle of attack $\alpha$, for the pulsed blowing application with $\mathrm{C}_{\mu} \leq 0.5 \%$. In figure 3 , for the low $\alpha \approx 0^{\circ} \ldots 4^{\circ}$, the reference lift curve is actually translated to the left when AFC is activated, with different increments, depending on $\mathrm{C}_{\mu}$. With the increase of $\alpha$ a positive increment is computed even for the maximum lift ( $\sim 15 \mathrm{lc})$, with a corresponding reduction of the maximum angle of attack of about $2^{\circ}$. These global lift enhancements are resulting from the surface integration for the complete high-lift model, main wing and flap. The time-averaged local pressure distribution and skin friction is plotted in figure 4 for a fixed angle of attack, $\alpha=4^{\circ}$. First, on the left side graph, the pulsed blowing on the flap results in comparison to the reference in lower pressure levels for the flap suction peak, main wing trailing edge as well as for the main wing suction peak. Second, on the right side figure, the skin friction distribution for the flap shows the reduction of the separation length by AFC; the larger the $\mathrm{C}_{\mu}$ the smaller is the separation length. The flowfield in the flap vicinity, as shown figure 5, is the final evidence of the massive reduction of the flap separation with AFC. While from the pressure distribution it is hard to extract an evidence for the existence of the flap separation in the time average data (here as e.g. at $\mathrm{C} \mu=0.5 \%$ ), the computed mean flowfield data is a welcomed indicator for the evaluation of the AFC application.

A relevant aspect for the numerical simulation is the verification and validation with experimental data and belongs to one of the challenges for the unsteady AFC simulations. For the numeric it is a challenge with respect to the turbulence modeling, the definition of the actuation inflow conditions as well as the modeling requirements for a specific model setup in a windtunnel; for the experiment it is a challenge to generate unsteady data with high accuracy, to ensure repeatability and independence of windtunnel influence as well to precisely define the existing flow conditions at the actuator. Here, the comparison CFD with experiment is limited to 
the time-averaged pressure distributions under a comparable AFC setup, and serve only for a qualitative evaluation. In figure 6 the pressure distributions with and without $\mathrm{AFC}$ are depicted for a fixed moderate angle of attack, the lines correspond to CFD results, while the symbols belong to experimental data. The computed trends of the AFC application are in good agreement with the experimental observations for both the main wing and the flap pressure distributions. As in the experiments at low $\mathrm{C}_{\mu}$ the flap separation is reduced but not suppressed with a global lift enhancement of the order of $10 \mathrm{lc}$.

Here, for the geometrical setup of interest, the influence of 2 classical actuation parameters, blowing momentum and frequency, is further discussed based on the numerical results. Not only that at very low $\mathrm{C}_{\mu}$ none, or just limited enhancement is computed, but the efficiency charts depict a saturation level at the higher $\mathrm{C}_{\mu}$ (figure 7). This is the result of minimizing the flap separation (as shown previously for $\alpha=4^{\circ}$ ) with the consequent higher flap suction peak and in the same time reaching a main wing trailing edge pressure which may be further decreased only by high additional $\mathrm{C}_{\mu}$. The lift enhancement of the order of $50 \mathrm{lc}$ is a result of the surface integration as sum of about $40 \mathrm{lc}$ from the main wing and about $10 \mathrm{lc}$ from the flap. The actuation setup was design and has proved an efficient separation control by pulsed blowing and, in the same time, the blowing direction, $45^{\circ}$ downstream, is relatively inappropriate for further circulation control likewise to an expected result for a tangential constant blowing. An example is shown in figure 8, where the pressure distributions and skin friction distributions for the flap at $\mathrm{C}_{\mu}=0.3 \%$ and $\mathrm{C}_{\mu}=0.5 \%$ depict a very low benefit of the higher blowing momentum after the flap separation was already suppressed at $\mathrm{C}_{\mu}=0.3 \%$.

The second parameter study is for the actuation frequency in the range of $\mathrm{F}^{+}=0.16 \ldots 0.64$. In general, the numerical results indicate a minor impact of the frequency on the aerodynamic performance as exemplified in figure 9. On the right side of the graph the time-averaged lift coefficients show a relative constant lift for $\mathrm{F}^{+}=0.16 \ldots 0.48$, and a small decrease at the highest computed frequency $\mathrm{F}^{+}=0.64$. Except the mean values, the airfoil lift variation over time suggests a maximum global load variation for the $\mathrm{F}^{+}=0.16$ and a corresponding minimum for the $\mathrm{F}^{+}=0.64$. The load variation is triggered directly by the flow topology changes in time at the trailing edge flap. The large coherent structures, giving the pulsed excitation mechanism, lead only at low frequency to large changes over time in the local pressure field, and as shown in figure $10\left(\mathrm{~F}^{+}=0.16\right)$ to noticeable changes in the separation topology from one state to the other. For all the other three frequencies the computations indicate deviations of maximum $\pm 2.5 \mathrm{lc}$ from the mean value of the lift coefficient and as expected the flow topology changes over time are very restricted (not shown here).

Furthermore it was of interest to check the applicability of this AFC concept for high Reynolds numbers. The simulations show for the design point $\left(\mathrm{Re}=7 \times 10^{6}\right)$ a similar lift enhancement with the increase of blowing momentum coefficient as in the low Reynolds number test case, where lift increments of the order of $17 \%$ are 
reached for the $\mathrm{C}_{\mu}=0.3 \%$ (figure 11 ). The flap topology depicted in figure 12 shows the successful separation reduction by $\mathrm{AFC}$, while the saturation point (attached flow on the flap) is not reached at $\mathrm{C}_{\mu}=0.3 \%$. Yet it is evident the higher flap suction peak with AFC and the global resulting enhancement.

\section{Conclusions}

URANS simulations concerning local pulsed excitation on a high-lift airfoil were performed focusing on the parameter study for the blowing momentum and frequency. For moderate momentum coefficient $\mathrm{C}_{\mu}$, global lift enhancements of up to 50 lc have been computed while the large flap separation was massively reduced. Frequency changes in the range of $\mathrm{F}^{+}=0.16 \ldots 0.64$ had a low impact on the mean aerodynamic performance, while the loads variation over time suggested that the higher the frequency the smaller is the lift variation over time; the computed $\mathrm{F}^{+}=0.48$ may be considered as global optimum. Except the parameter study at low Reynolds number the applicability of this AFC technique was also shown for the airfoil design point $\mathrm{Re}=7 \times 10^{6}$.

\section{Acknowledgment(s)}

The work was partially supported by the EU-project AVERT in the European Sixth Framework Programme, contract no. AST5-CT-2006-030914.

\section{References}

[1] Petz, R., Nitsche, W.: Active Separation Control on the Flap of a Two-Dimensional Generic High-Lift Configuration, Journal of Aircraft 2007, 0021-8669 vol.44 no.3 (865874).

[2] Bauer, M., Peltzer, I., Nitsche, W., Goelling, B.: Active Flow Control on an IndustryRelevant Civil Aircraft Half Model, In Active Flow Control II, vol. 108 of Notes of Numerical Fluid Mechanics and Multidisciplinary Design, 2010 (95-107).

[3] Ciobaca, V.: Simulation of Active Flow Control on the Flap of a 2D High-Lift Configuration, In "New Results in Numerical and Experimental Fluid Mechanics VII", vol. 112 of Notes on Numerical Fluid Mechanics and Multidisciplinary Design (NNFM), 2010.

[4] T. Naveh, A. Seifert, A. Tumin, I. Wygnanski, Sweep effect on the parameters governing the control of separation by periodic excitation, J. Aircraft 35 (3) (1998) 510 512.

[5] A. Seifert, T. Bachar, D. Koss, M. Shepshelovich, I. Wygnanski, Oscillatory blowing, a tool to delay boundary layer separation, AIAA J. 31 (11) (1993) 2052-2060.

[6] Wild, J., Wichmann, G., Haucke, F, Peltzer, I, Scholz, P.: Large scale separation flow control experiments within the German Flow Control Network, AIAA-2009-530.

[7] Wild, J., Pott-Pollenske, M., and Nagel, B.: An Integrated Design Approach for Low Noise Exposing High-Lift Devices, AIAA-2006-2843. 


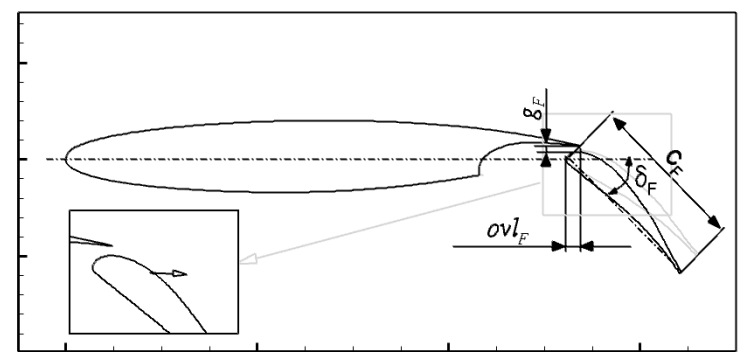

Figure 1: F15 high-lift configuration.
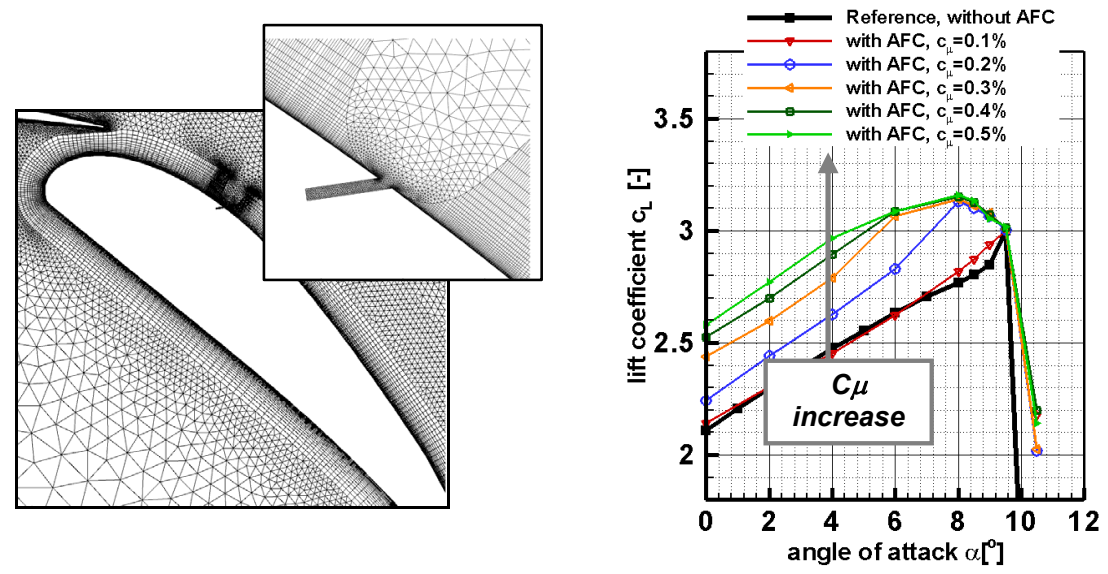

Figure 2: Unstructured grid in the flap Figure 3: Lift curves at $\operatorname{Re} \approx 2 \times 10^{6}$ (AFC: vicinity. $\mathrm{F}+=0.32$ ).
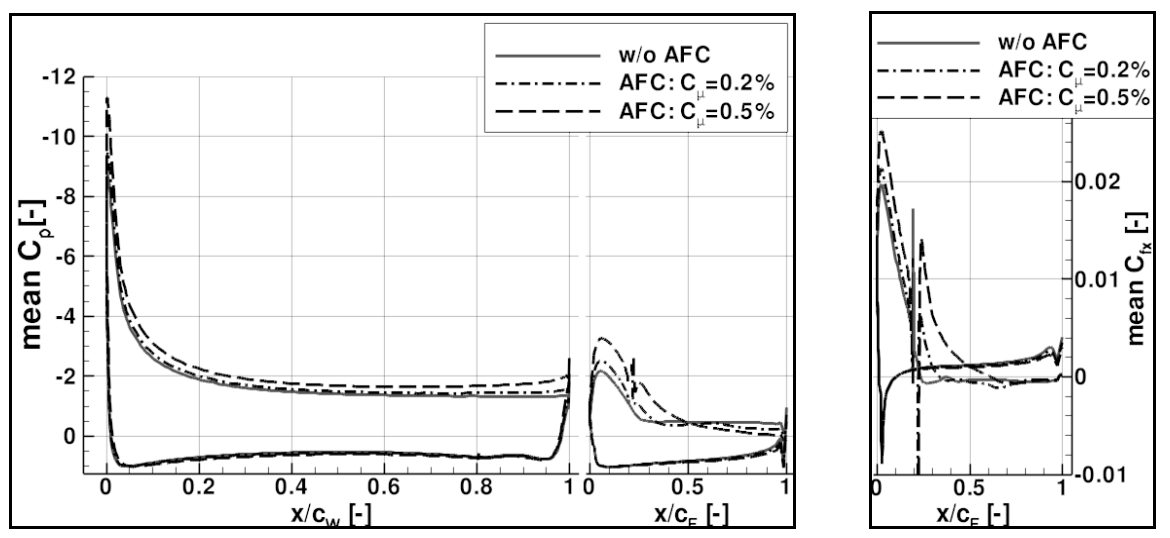

Figure 4: Pressure coefficient and friction coefficient distributions for $\alpha=4^{\circ}$ at $\operatorname{Re} \approx 2 \times 10^{6}$ (AFC: $\mathrm{F}+=0.32$ ). 

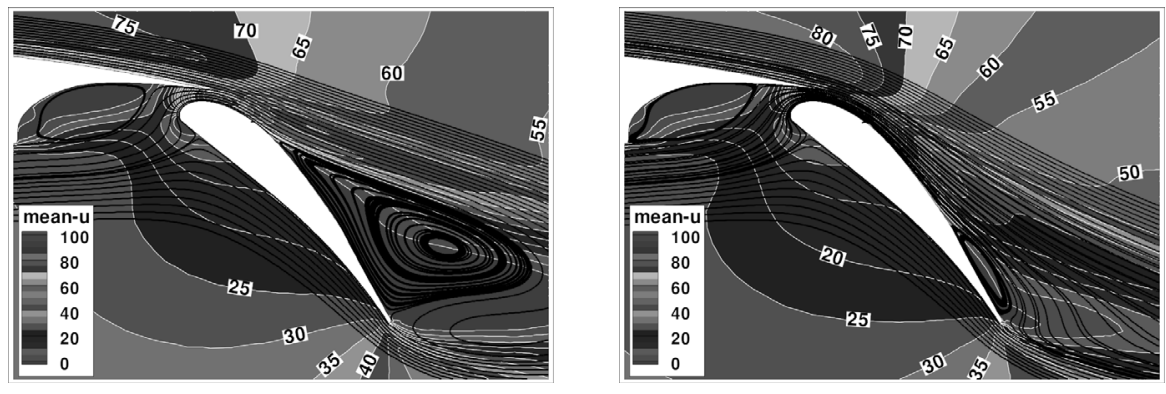

Figure 5: Mean flow streamlines and mean streamwise velocity distributions for $\alpha=4^{\circ}$ at $\mathrm{M}=0.15\left(\mathrm{U}_{\infty} \approx 50 \mathrm{~m} / \mathrm{s}\right), \operatorname{Re} \approx 2 \times 10^{6}$; left: w/o AFC, right: with AFC $(\mathrm{F}+=0.32, \mathrm{C} \mu=0.5 \%)$.

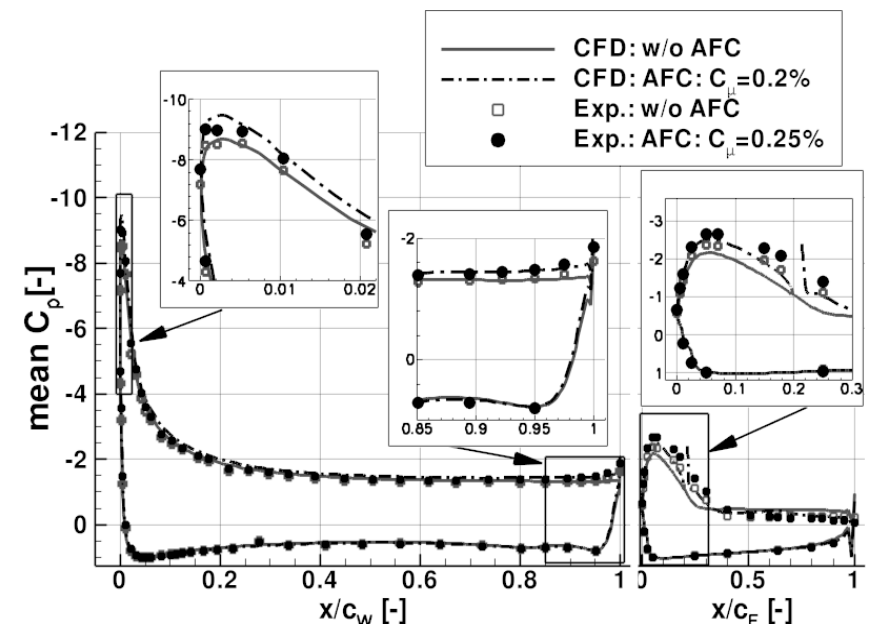

Figure 6: Comparison CFD vs. Experiment for the pressure coefficient distributions with and without $\mathrm{AFC}$ at $\mathrm{M} \approx 0.15, \mathrm{Re} \approx 2 \times 10^{6}$.

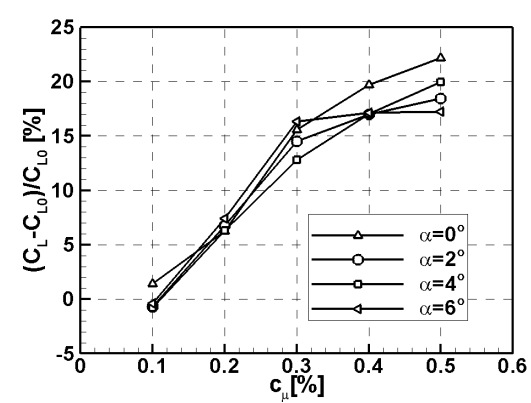

AFC: $\mathrm{F}+=0.32 \& \mathrm{C}_{\mathrm{L} 0}: \mathrm{w} / \mathrm{o} \mathrm{AFC}$

Figure 7: AFC efficiency at $\operatorname{Re} \approx 2 \times 10^{6}$.

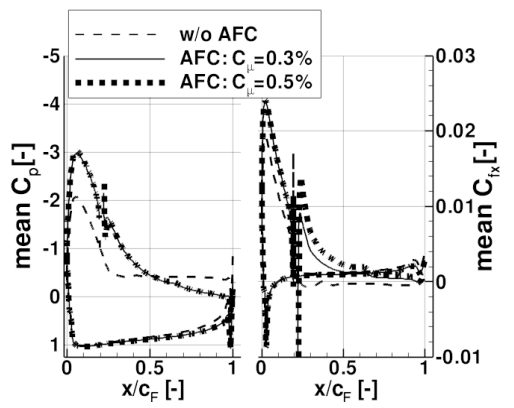

AFC: $F+=0.32 \& \alpha=6^{0}, \operatorname{Re} \approx 2 \times 10^{6}$

Figure 8: Flap topology with \& w/o AFC. 


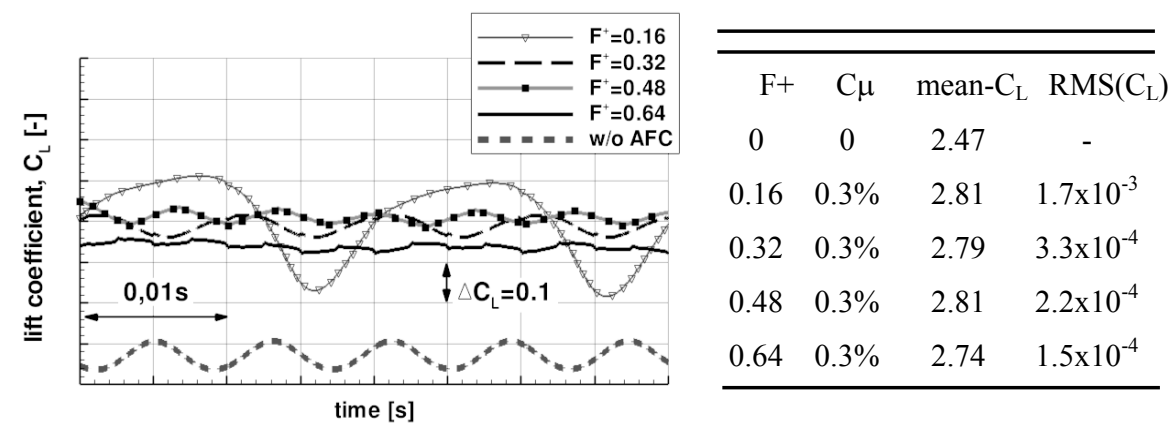

Figure 9: Global lift coefficient variation over time for various frequencies $\left(\mathrm{C} \mu=0.3 \%, \alpha=4^{\circ}\right)$ and the resulting mean lift coefficients.
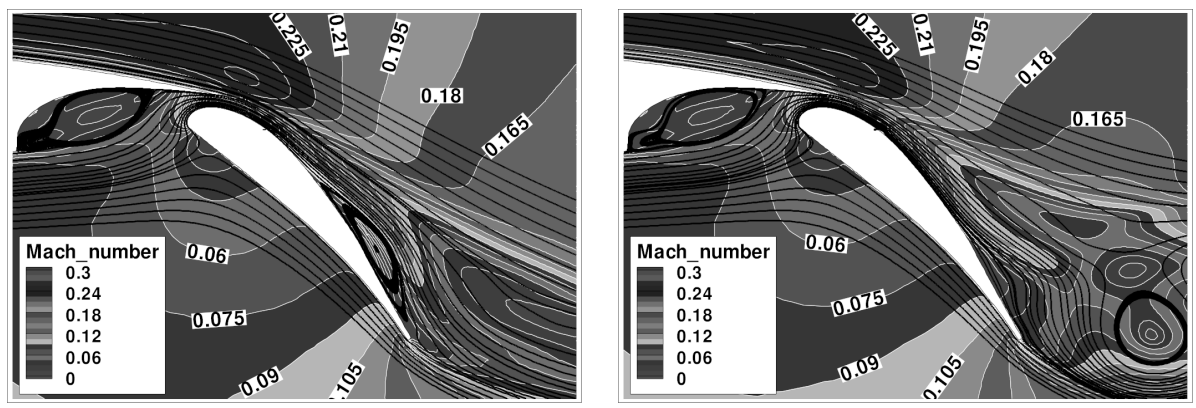

Figure 10: Instantaneous flow streamlines and Mach number distributions for $\alpha=4^{0}$ at $\mathrm{M}=0.15, \mathrm{Re} \approx 2 \times 10^{6}$; with $\mathrm{AFC}(\mathrm{F}+=0.16, \mathrm{C} \mu=0.3 \%)$; left $\mathrm{t}=\mathrm{t}_{1}\left(\min \mathrm{C}_{\mathrm{L}}\right)$, right $\mathrm{t}=\mathrm{t}_{1}+0.012 \mathrm{~s}$ $\left(\max C_{L}\right)$.

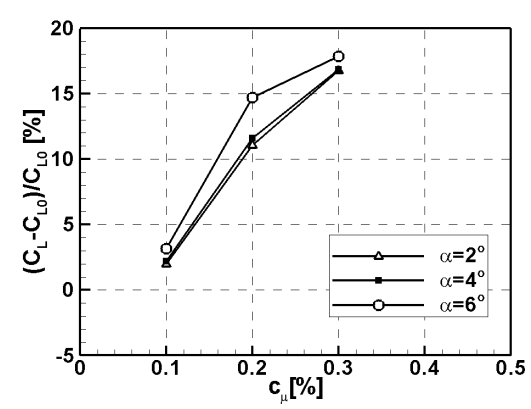

AFC: $\mathrm{F}+=0.32 \& \mathrm{C}_{\mathrm{L} 0}: \mathrm{w} / \mathrm{o}$ AFC

Figure 11: AFC efficiency at $\operatorname{Re} \approx 7 \times 10^{6}$.

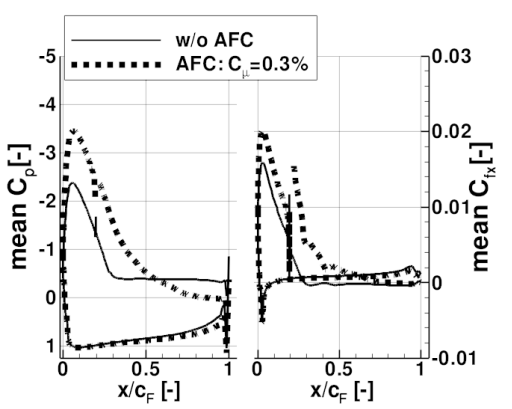

AFC: $F+=0.32 \& \alpha=6^{0}, \operatorname{Re} \approx 7 \times 10^{6}$

Figure 12: Flap topology with \& w/o AFC. 